\title{
A NOTE ON EXPONENTIALLY HARMONIC MORPHISMS
}

\author{
ERIC LOUBEAU \\ Université de Bretagne Occidentale, UFR Sciences et Techniques, Departement de Mathematiques, \\ 6, Avenue Victor Le Gorgeu, BP 809, 29285 Brest Cedex, France \\ e-mail:loubeau@univ-brest.fr \\ and STEFANO MONTALDO \\ University of Leeds, Department of Pure Mathematics, LS2 9JT Leeds, UK \\ e-mail:pmtms@amsta.leeds.ac.uk
}

(Received 24 March, 1998)

\begin{abstract}
We prove that exponentially harmonic morphisms are precisely the Riemannian submersions with minimal fibres.

1991 Mathematics Subject Classification. 58E20
\end{abstract}

1. Introduction. Let $(M, g)$ and $(N, h)$ be two Riemannian manifolds, and $\phi: M \rightarrow N$ a smooth map. For any compact domain $\Omega \subseteq M$ define the exponentialenergy of $\phi$ (cf. [3]) by

$$
\mathbb{E}(\phi)=\int_{\Omega} \exp (e(\phi)) v_{g}
$$

where $e(\phi)=\frac{1}{2}\|d \phi\|^{2}$ is the energy density of $\phi$ and $v_{g}$ the Riemannian volume element. A smooth map $\phi:(M, g) \rightarrow(N, h)$ is an exponentially harmonic map if it is an extremal of the exponential-energy $\mathbb{E}$ for any compact domain $\Omega \subseteq M$.

The Euler-Lagrange equation of this problem can be written (cf. [3]) as

$$
t(\phi)=\operatorname{div}(\exp e(\phi) d \phi)=\exp e(\phi)(\tau(\phi)+d \phi \operatorname{grad} e(\phi))=0,
$$

where $\tau$ is the usual tension field given by $\tau(\phi)=\operatorname{div}(d \phi)$. We recall for further use that a map satisfying $\tau(\phi)=0$ is a harmonic map; i.e. a critical point of the energy $E(\phi)=\int_{M} e(\phi) v_{g}(\mathrm{cf} .[4])$.

An exponentially harmonic morphism is a smooth map $\phi:(M, g) \rightarrow(N, h)$ which pulls back local exponentially harmonic functions to exponentially harmonic functions.

The aim of this note is to prove the following result.

Theorem 1.1. A smooth map $\phi:(M, g) \rightarrow(N, h)$ is an exponentially harmonic morphism if and only if it is a Riemannian submersion with minimal fibres.

\section{Preliminaries.}

2.1 Horizontal weak conformality. Let $\phi:(M, g) \rightarrow(N, h)$ be a smooth map between two Riemannian manifolds. The tangent space at a point $x \in M$ can be decomposed as $T_{x} M=H_{x} \oplus V_{x}$, where $V_{x}=\operatorname{ker}\left(d \phi_{x}\right)$ and $H_{x}=V_{x}{ }^{\perp}$. The spaces $V_{x}$ and $H_{x}$ are called the vertical and horizontal spaces at the point $x \in M$ respectively.

Let $C_{\phi}=\left\{x \in M \mid \operatorname{rank}\left(d \phi_{x}\right)\right.$ is not maximal $\}$ be the set of critical points of $d \phi$. 
Definition 2.1. A map $\phi:(M, g) \rightarrow(N, h)$ is called horizontally weakly conformal if, for every $x \in M \backslash C_{\phi},\left.d \phi_{x}\right|_{H_{x}}$ is conformal and surjective.

If $\phi:(M, g) \rightarrow(N, h)$ is horizontally weakly conformal, then there exists a function $\lambda: M \backslash C_{\phi} \longrightarrow \mathbb{R}^{+}$such that $\lambda^{2} g_{x}(X, Y)=h_{\phi(x)}\left(d \phi_{x}(X), d \phi_{x}(Y)\right)$, for all $X, Y \in H_{x}$, and $x \in M \backslash C_{\phi}$. The function $\lambda$ can be extended continuously to the whole of $M$ by setting $\left.\lambda\right|_{C_{\phi}}=0$. The extended function is called the dilation function of $\phi$.

Note that $\lambda^{2}$ is smooth.

REMARK 2.2. If $d \phi(\operatorname{grad} e(\phi))=0$, then $\phi$ is exponentially harmonic if and only if it is harmonic. If $\phi$ is horizontally weakly conformal, then $e=\frac{n}{2} \lambda^{2}$, and the condition $d \phi(\operatorname{grad} e(\phi))=\frac{n}{2} d \phi\left(\operatorname{grad} \lambda^{2}\right)=0$ means that $\phi$ is horizontally homothetic; i.e. $\operatorname{grad}\left(\lambda^{2}\right)$ is vertical.

A Riemannian submersion is a horizontally weakly conformal map with $\lambda^{2}=1$. Riemannian submersions are harmonic maps if and only if the fibres are minimal submanifolds (cf. [4]), and from Remark 2.2, they are exponentially harmonic if and only if the fibres are minimal submanifolds.

2.2 Existence of exponentially harmonic functions. Throughout the rest of this article, we assume the Einstein convention on the summation of repeated indices.

Proposition 2.3. Let $\left(N^{n}, h\right)$ be a Riemannian manifold. Then, for any point $q \in N$ and any set of constants $\left\{C_{\alpha \beta}\right\}\left(C_{\alpha \beta}=C_{\beta \alpha}\right),\left\{C_{\alpha}\right\}$ where $\alpha, \beta \in\{1, \ldots, n\}$, satisfying

$$
\sum_{\alpha} C_{\alpha \alpha}+\sum_{\beta, \delta} C_{\beta} C_{\delta} C_{\beta \delta}=0
$$

there exists an exponentially harmonic function $f$ defined on a neighbourhood $U \subseteq N$ of $q$ such that in a system of normal local coordinates $\left(y^{\alpha}\right)$ centred on $q$ we have

$$
\begin{aligned}
\frac{\partial^{2} f}{\partial y^{\alpha} \partial y^{\beta}}(q) & =C_{\alpha} \beta, \\
\frac{\partial f}{\partial y^{\alpha}}(q) & =C_{\alpha} .
\end{aligned}
$$

Proof. The exponential tension field $\mathrm{t}$ is an elliptic operator, though not uniformly (cf. [3]). Moreover t is quasi-linear (as defined in [5]) since for a map $\phi$ from a manifold $(M, g)$ equipped with a system of coordinates $\left(x^{i}\right)_{i=1, \ldots, \operatorname{dim} M}$ to a manifold $(N, h)$ equipped with $\left(y^{\alpha}\right)_{\alpha=1, \ldots, \operatorname{dim} N}$, the terms of highest order, i.e. of order two, of $\mathrm{t}(\phi)$ are

$$
\exp e(\phi)\left(g^{i j} \frac{\partial^{2} \phi^{\alpha}}{\partial x^{i} \partial x^{j}}+g^{i j} h_{\delta \gamma} g^{k l} \frac{\partial \phi^{\alpha}}{\partial x^{i}} \frac{\partial \phi^{\gamma}}{\partial x^{l}} \frac{\partial^{2} \phi^{\delta}}{\partial x^{k} \partial x^{j}}\right)
$$

which are linear in the second derivatives of the map $\phi$.

Proposition 2.3 is then an application of [1, Theorem 2.4], which basically states that, for quasi-linear elliptic operators, infinitesimal solvability, i.e. Condition (2.1), implies local solvability. 


\section{Characterisation.}

Proposition 3.1. (Composition Law). Let $\phi:(M, g) \rightarrow(N, h)$ and $\psi:(N, h) \rightarrow(P, k)$ be two maps between Riemannian manifolds. Then the exponential tension field of the composition $\psi \circ \phi$ has the form

$$
\begin{aligned}
\mathrm{t}(\psi \circ \phi) & =\exp e(\psi \circ \phi)(d \psi(\tau(\phi))+\operatorname{trace}(\nabla d \psi)(d \phi, d \phi) \\
& \left.+\frac{1}{2} \theta^{2} d(\psi \circ \phi) \operatorname{grad}\|d \phi\|^{2}+\frac{1}{2}\|d \phi\|^{2} d(\psi \circ \phi) \operatorname{grad} \theta^{2}\right),
\end{aligned}
$$

where $\theta=\frac{|d(\psi \circ \phi)|}{|d \phi|}$.

Proof. This follows from the equations

$$
\operatorname{grad}\|d(\psi \circ \phi)\|^{2}=\operatorname{grad}\left(\frac{\|d(\psi \circ \phi)\|^{2}}{\|d \phi\|^{2}}\|d \phi\|^{2}\right)=\|d \phi\|^{2} \operatorname{grad} \theta^{2}+\theta^{2} \operatorname{grad}\|d \phi\|^{2}
$$

and the usual composition law of the tension field:

$$
\tau(\psi \circ \phi)=d \psi(\tau(\phi))+\operatorname{trace}(\nabla d \psi)(d \phi, d \phi) .
$$

Proposition 3.2. Let $\phi:(M, g) \rightarrow(N, h)$ be a Riemannian submersion. Then $\phi$ is an exponentially harmonic morphism if and only if $\phi$ is harmonic.

Proof. Let $f:(N, h) \rightarrow \mathbb{R}$ be a function. From the composition law we have

$$
\begin{aligned}
\mathrm{t}(f \circ \phi) & =\exp e(f \circ \phi)(d f(\tau(\phi))+\operatorname{trace}(\nabla d f)(d \phi, d \phi) \\
& \left.+\frac{1}{2} \theta^{2} d(f \circ \phi) \operatorname{grad}\|d \phi\|^{2}+\frac{1}{2}\|d \phi\|^{2} d(f \circ \phi) \operatorname{grad} \theta^{2}\right),
\end{aligned}
$$

where $\theta=\frac{|d(f \circ \phi)|}{|d \phi|}$.

Using the fact that $\phi: M \rightarrow N^{n}$ is a Riemannian submersion we obtain

$$
\begin{gathered}
\operatorname{grad}\|d \phi\|^{2}=0, \quad \theta^{2}=\frac{\|d f\|^{2}}{n}, \\
\frac{\|d \phi\|^{2}}{n}=1, \quad \operatorname{trace}(\nabla d f)(d \phi, d \phi)=\tau(f) \circ \phi .
\end{gathered}
$$

From these equalities the composition law becomes

$$
\mathrm{t}(f \circ \phi)=\exp e(f) d f(\tau(\phi))+\mathrm{t}(f) \circ \phi .
$$

If $\phi$ is harmonic we have

$$
\mathrm{t}(f \circ \phi)=t(f) \circ \phi
$$


which implies that $\phi$ is an exponentially harmonic morphism. Conversely, if $\phi$ is an exponentially harmonic morphism, then for any exponentially harmonic function $f$ we have

$$
d f(\tau(\phi))=0
$$

which implies that $\tau(\phi)=0$.

Proposition 3.3. Let $\phi:(M, g) \rightarrow(N, h)$ be a non-constant exponentially harmonic morphism. Then $\phi$ is a Riemannian submersion.

Proof. For a given point $x \in M^{m}$, we equip $\left(M^{m}, g\right)$ with a system of normal coordinates $\left(x^{i}\right)_{i=1, \ldots, m}$ around $x$ and $\left(N^{n}, h\right)$ with normal coordinates $\left(y^{\alpha}\right)_{\alpha=1, \ldots, n}$ centred on $\phi(x)$. In the sequel we write $f_{\alpha}$ for $\frac{\partial f}{\partial y^{\alpha}}$ and $\phi_{i}^{\alpha}$ for $\frac{\partial \phi^{\alpha}}{\partial x^{i}}$. In normal coordinates the exponential harmonic tension field is

$$
\mathrm{t}^{\alpha}(\phi)=\phi_{i i}^{\alpha}+\sum_{\beta} \phi_{i}^{\alpha} \phi_{j}^{\beta} \phi_{i j}^{\beta}
$$

Let $f: N \rightarrow \mathbb{R}$ be an exponentially harmonic function in a neighbourhood of $\phi(x)$. Since $\phi$ is an exponentially harmonic morphism we have

$$
\mathrm{t}(f \circ \phi)=f_{\alpha \beta} \phi_{i}^{\beta} \phi_{i}^{\alpha}+f_{\alpha} f_{\beta} f_{\gamma \delta} \phi_{i}^{\alpha} \phi_{j}^{\beta} \phi_{i}^{\gamma} \phi_{j}^{\delta}+f_{\alpha} \phi_{i i}^{\alpha}+f_{\alpha} f_{\beta} f_{\gamma} \phi_{i}^{\alpha} \phi_{j}^{\beta} \phi_{i j}^{\gamma}=0 .
$$

Proposition 2.3 implies, for each $\gamma \in(1, \ldots, n)$ the existence of an exponentially harmonic function $f$ such that at $\phi(x), \frac{\partial^{2} f}{\partial y^{\alpha} \partial y^{\beta}}=0$ and $\frac{\partial f}{\partial y^{\alpha}}=\delta_{\alpha \gamma}$. At $x,(3.1)$ implies that

$$
\phi_{i i}^{\gamma}+\phi_{i}^{\gamma} \phi_{j}^{\gamma} \phi_{i j}^{\gamma}=0 \text {. }
$$

We apply Proposition 2.3 again, this time with $C_{\alpha}=0$ and any $C_{\alpha \beta}=C_{\beta \alpha}$ such that $\sum C_{\alpha \alpha}=0$, and we deduce from (3.1) that

$$
C_{\alpha \beta} \phi_{i}^{\beta} \phi_{i}^{\alpha}=0 \text {. }
$$

This condition implies in a standard way (cf. [2, p. 42]) that $\phi$ is horizontally weakly conformal; i.e. $\phi_{i}^{\beta} \phi_{i}^{\alpha}=\lambda^{2} \delta^{\alpha \beta}$. Using the horizontal conformality, (3.1) becomes

$$
\lambda^{2} f_{\alpha \alpha}+\lambda^{4} f_{\alpha} f_{\beta} f_{\alpha \beta}+f_{\alpha} \phi_{i i}^{\alpha}++f_{\alpha} f_{\beta} f_{\gamma} \phi_{i}^{\alpha} \phi_{j}^{\beta} \phi_{i j}^{\gamma}=0
$$

Using Proposition 2.3 again, with $C_{\alpha}=0, \alpha \neq \alpha_{0}, C_{\alpha_{0}}=1$ and any $C_{\alpha \beta}=C_{\beta \alpha}$ such that $\sum C_{\alpha \alpha}+C_{\alpha_{0} \alpha_{0}}=0$, we obtain

$$
\lambda^{2} f_{\alpha \alpha}\left(1-\lambda^{2}\right)+\phi_{i i}^{\alpha_{0}}+\phi_{i}^{\alpha_{0}} \phi_{j}^{\alpha_{0}} \phi_{i j}^{\alpha_{0}}=0
$$

Also, using (3.2), we finally have 


$$
\lambda^{2} f_{\alpha \alpha}\left(1-\lambda^{2}\right)=0
$$

which implies $\lambda^{2}=1$ and thus $\phi$ is a Riemannian submersion.

Combining Proposition 3.2 and Proposition 3.3 yields Theorem 1.1.

ACKNOWLeDGements. The second author would like to thank the Département de Mathématiques at the Université de Bretagne Occidentale, Brest, for making possible a visit in February 1998 where much of this work was done.

\section{REFERENCES}

1. S. Alinhac and P. Gerard, Opérateurs pseudo-differentiels et théorème de Nash-Moser, Savoirs Actuels (InterEditionsEditions du C.N.R.S., 1991).

2. J. Eells and L. Lemaire, Selected topics in harmonic maps, CBMS Regional Conf. Ser. in Math. 50 (Amer. Math. Soc., Providence, R.I., 1983).

3. J. Eells and L. Lemaire, Some properties of exponentially harmonic maps, Banach Center Publ. 27 (1992), 129-136.

4. J. Eells and J. H. Sampson, Harmonic mappings of Riemannian manifolds, Amer. J. Math. 86 (1964), 109-160.

5. D. Gilbarg and N. S. Trudinger, Elliptic partial differential equations of second order, A Series of Comprehensive Studies in Mathematics 224 (Springer-Verlag, 1977). 